\title{
INFLUÊNCIA DA REALIDADE VIRTUAL NAS ATIVIDADES PSICOMOTORAS E PERCEPÇÃO CORPORAL DE ESCOLARES: ESTUDO PILOTO
}

\author{
INFLUENCE OF VIRTUAL REALITY IN PSYCHOMOTOR ACTIVITIES AND BODY PERCEPTION OF \\ SCHOOL STUDENTS: A PILOT STUDY
}

\section{Elenilton Correia de Souza ${ }^{a^{*}}$, Ana Paula de Lima Ferreira ${ }^{b^{*}}$}

\author{
aelenilton2010@gmail.com, bapllima@yahoo.com.br \\ *Universidade Federal de Sergipe - Aracaju (SE), Brasil \\ **Universidade Federal de Pernambuco - Recife (PE), Brasil
}

Data de recebimento do artigo: 13/05/2015

Data de aceite do artigo: 06/10/2015

\section{RESUMO}

Introduçáo: A psicomotricidade pode ser definida como a ciência que trabalha de forma associada o corpo e a mente. Recursos de realidade virtual podem ser ferramentas lúdicas para estimular a aquisição e o aperfeiçoamento de habilidades neuromotoras em crianças em fase de desenvolvimento. Objetivo: analisar a influência do Nintendo Wii ${ }^{\circledR}$ nas atividades psicomotoras e na percepção corporal de crianças saudáveis da rede pública de ensino. Materiais e métodos: Foram envolvidos nessa amostra aleatória três estudantes de ambos os gêneros, na faixa etária de 7 a 10 anos. A percepção corporal foi avaliada através do Protocolo de Askevold, o equilíbrio foi verificado pelo teste de Fukuda e o desempenho de práticas psicomotoras foi analisado por meio do protocolo de teste psicomotor de Fonseca. Após avaliaçóes, as crianças passaram por um treinamento com a realidade virtual duas vezes por semana durante 30 minutos, totalizando 10 sessóes. Para analisar os resultados obtidos foram considerados valores absolutos e relativos. Resultados: Referente ao COG Wii Fit e estabilidade pôde acompanhar ganhos do momento pré para o pós. As pontuaçôes aumentaram praticamente em todos os jogos utilizados na intervenção. Além disso foi verificada melhorias importantes na percepção corporal dos estudantes, ou seja, saindo de uma condição de hiperesquematia para normoesquematia. Conclusáo: Pôde-se constatar que o treinamento com a realidade virtual influenciou positivamente o desempenho nas atividades psicomotoras e percepção corporal de todas as crianças analisadas.

Palavras-chave: Jogos virtuais; noção corporal; psicomotricidade; crianças.

\section{ABSTRACT}

Introduction: Psychomotricity can be defined as the science that works on the connection of body and mind. Virtual reality features can be playful tools to stimulate neuromotor abilities acquisition and improvement in growing children. Objective: To analyze Nintendo Wii influence on psychomotor activities and body awareness in healthy children the public school system. Materials and methods: We randomly involved in this sample three students of both genders, aged 7-10 years. Body perception was evaluated by the Askevold Protocol, balance was verified by the Fukuda test and psychomotor practices performance was analyzed by the Fonseca psychomotor test protocol. After evaluations, children have gone through a two-times-a-week virtual reality training for 30 minutes each, in a total of 10 sessions. Absolute and relative values were considered to analyze the results. Results: Regarding the COG Wii Fit ${ }^{\oplus}$ and stability could follow gains from prior to after the intervention. Score has increased in almost all games used in the intervention. Important improvements were also observed in students body perception, changing hiperesquematia condition for normoesquematia condition. Conclusion: It could be observed that virtual reality training has positively influenced performance on psychomotor activities and body perception in all children studied.

Keywords: Virtual games; body notion; psychomotricity; children. 


\section{Introdução}

A psicomotricidade é uma ciência que trabalha de forma interligada com o corpo e a mente. É através dela que o indivíduo vai aperfeiçoando seus movimentos e tornando-os mais capacitados para a realização de tarefas diárias. Além disso, sabe-se que os aspectos psicomotores estão relacionados com as atividades designadas durante o processo de aquisiçáo motora em crianças, promovendo a geração de uma série de conhecimentos e domínios indispensáveis. Isso possibilita que o corpo em fase de crescimento seja controlado mediante às exigências biomecânicas ${ }^{1}$. Paralelamente a esses mecanismos, ainda é fundamental a associação de inúmeros fatores como os ambientais, físicos, sociais, psicológicos ${ }^{2}$, culturais, familiares e características individuais de cada criança ${ }^{3}$.

Entre os aspectos psicomotores que precisam estar bem desenvolvidas na primeira infância destacam-se: (a) a tonicidade; (b) a estruturação espaço-temporal; (c) a lateralidade; (d) o equilíbrio; (e) a praxia global; (f) a praxia fina e $(\mathrm{g})$ a noçáo corporal. Se as crianças forem bem estimuladas, utilizarão esses aspectos psicomotores com uma frequência que irá contribuir na aquisição das habilidades envolvendo o reconhecimento de tempo, espaço, formulaçóes de conceitos e ideias que resultarão em experiências neuropsicomotoras ${ }^{4}$.

Para Almeida ${ }^{2}$, o adequado desenvolvimento psicomotor está associado ao processo de ensino-aprendizagem. Para todas as atividades motoras existe uma associação entre a mente e o corpo, sendo que falhas nessa integração podem contribuir para o surgimento de problemas da aprendizagem, postura, direção, lateralidade e ritmo ${ }^{5}$. Entre as principais atividades motoras destacam-se o andar, chutar, arremessar, agarrar e lançar. Para que tais habilidades sejam realizadas é essencial que os sistemas que integram o equilíbrio estejam preservados ${ }^{6}$. Penchiná et al. ${ }^{7}$ apontam a importância da participação efetiva do sistema nervoso (SN) durante o desenvolvimento neuropsicomotor, uma vez que o $\mathrm{SN}$ atua na geração das capacidades funcionais, comportamento e aprendizagem. Nesse processo são imprescindíveis uma série de informaçóes provenientes do sistema vestibular, dos receptores visuais e do sistema somatosensorial.

Lemos et al. ${ }^{8}$, aponta que as oscilaçóes corpóreas que geram o desequilíbrio ou instabilidade podem estar intimamente relacionadas a alteraçôes no CG (Centro de Gravidade), bem como a quantidade de massa corporal. O equilíbrio faz parte da motricidade humana, sendo um componente essencial para gerar uma adequada postura estática e dinâmica, podendo ser testado através de testes estabilométricos ${ }^{9}$. O aperfeiçoamento do equilíbrio é desenvolvido junto da aquisição de experiências sensório-motoras diversas ${ }^{10}$.
Um outro componente de extrema importância e que está em plena construção desde a infância é o esquema corporal. Este é entendido como um modelo padrão da postura criado por cada indivíduo diante do espaço ao qual está inserido, servindo de alicerce básico para auxiliar durante as diferentes posturas e na execuçẫo dos movimentos corporais. Ter uma imagem corporal (IC) bem definida é fundamental para que as pessoas possam se localizar e movimentar-se adequadamente, uma vez que isso gera condições ideais para a realização das atividades diárias ${ }^{11}$. A IC corresponde a uma relação de autopercepção dos sujeitos diante de suas características individuais. Aspectos negativos referentes à IC se associam a sintomas depressivos, comprometimento de relacionamento social, baixa autoestima, ansiedade ${ }^{12}$ e distúrbios alimentares como a anorexia e bulimia ${ }^{13,14}$. Para Segheto et al. ${ }^{15}$, a percepção corporal além de referir-se ao reconhecimento dos membros diante do espaço ao qual estão situados, depende de uma associação dos mecanismos que envolvem os sistemas somatossensorial e vestibular.

Com isso, pode-se indagar que as crianças que não possuem uma consciência plena de seu corpo podem apresentar atrasos psicomotores, acarretando em deficiências funcionais durante a realizaçáo de movimentos multiplanares e multiarticulares. Recursos de tecnologia avançada como os videogames Nintendo Wii e $\mathrm{Xbox}^{\oplus}$ podem ser ferramentas adjuvantes no treinamento do esquema corporal de crianças em desenvolvimento. Contudo essa hipótese carece de investigaçóes; é preciso investigar se os jogos de realidade virtual (RV) podem potencializar estímulos para a prevenção de atrasos motores e alteraçóes de esquema corporal.

A escola é um ambiente natural que possibilita o fornecimento de estímulos psicomotores. Junto a ela podem ser agregados recursos tecnológicos para auxiliar no fornecimento de tais estímulos considerados fundamentais durante o desenvolvimento das crianças ${ }^{16}$.

A Wiiterapia mostra-se um recurso bastante promissor quando se trata de conceder estímulos ${ }^{17}$. Videogames como o Nintendo Wii ${ }^{\circledR}$ e a plataforma Wii $\mathrm{Fit}^{\circledR}$ podem apresentar efeitos satisfatórios quando são relacionados a dois fatores: os jogos escolhidos e a atraçáo natural que as crianças têm por objetos que trazem a ludicidade. As criança precisam ser motivadas e desafiadas para que aos poucos adquiram experiências oriundas dos erros. Tal ideia cabe adequadamente na proposta da realidade virtual ${ }^{2}$.

O Wii Fit ${ }^{\circledR}$ acoplado ao console Nintendo Wii ${ }^{\circledR}$ pode ser usado para detectar e melhorar a performance corporal através dos deslocamentos do peso na posição ortostática, ora pelas avaliaçóes corporais que a própria plataforma $\mathrm{faz}$, ora pelos jogos propriamente ditos ${ }^{17}$. O biofeedba$c k$ proporcionado pelos jogos possivelmente cria efeitos acumulativos de determinadas condiçóes ao longo do tempo. Além de ser algo divertido e prazeroso para as 
crianças, tal ferramenta pode ser usada para prevenção de atrasos cognitivos, melhora da consciência corporal, postura, coordenação motora, condicionamento físico e da qualidade de vida de uma forma geral ${ }^{18}$.

A Wiiterapia já vem sendo usada em alguns centros de reabilitaçáo neuromotora, mostrando-se um ferramenta importante no fornecimento de estímulos e aquisição de habilidades perdidas ou reduzidas, como por exemplo o equilíbrio corporal ${ }^{19}$. No entanto, é encontrada na literatura corrente uma proporção menor de estudos que mostram a utilização da realidade virtual no comportamento motor em crianças sadias em idade escolar. A utilização de videogames pode apresentar uma alternativa lúdica e auxiliar no processo de ensino-aprendizagem de crianças sadias. Além da parte cognitiva que é estimulada, as crianças também são desafiadas a desenvolverem habilidades fundamentais como equilíbrio, coordenação motora, agilidade e noção corporal. Portanto, o objetivo desse estudo foi analisar a influência da realidade virtual através de jogos do Nintendo Wii ${ }^{\circledR}$ nas atividades psicomotoras e na percepção corporal de escolares da rede pública de ensino.

\section{Casuística e métodos}

\section{Composição da amostra e considerações éticas}

Participaram desse estudo inicialmente 10 crianças, selecionadas aleatoriamente, na faixa etária de 7 a 10 anos de uma instituição de ensino público da cidade de Aracaju-SE. No entanto, para esse piloto foram selecionadas as três que passaram rigorosamente por todas as etapas da pesquisa. Previamente, os pais foram informados sobre o propósito da pesquisa, riscos e benefícios e, após aprovação da inclusão da criança na investigaçáo, os seus responsáveis assinaram um Termo de Consentimento Livre e Esclarecido (TCLE) conforme Resolução 466/12 do Conselho Nacional de Saúde, que trata o Código de Ética para Pesquisa em Seres Humanos. A pesquisa foi aprovada pelo Comitê de Ética em Pesquisa da Universidade Federal de Sergipe com o parecer CAAE no 174/2011.

\section{Critérios de inclusão e exclusão}

Foram incluídos os indivíduos que atendessem aos seguintes critérios de inclusão: crianças nascidas a termo, livres de complicações neurológicas e musculoesqueléticas, que apresentassem desenvolvimento cognitivo e psicomotor adequados para a faixa etária correspondente e as que praticassem com regularidade as aulas de educação física.

Foram adotados como critérios de exclusão nesse estudo crianças que possuíssem alguma deficiência motora e/ou cognitiva perceptível ou diagnosticada, aquelas que não realizassem nenhum tipo de atividade física, as que não recebessem autorização dos pais ou responsáveis ou ainda aquelas que faltassem a alguma das etapas da pesquisa.

\section{Tipo de estudo e local de realização da pesquisa}

Trata-se de um estudo piloto. As coletas de dados e as sessôes de realidade virtual aconteceram no laboratório de atividades práticas do curso de bacharelado em fisioterapia, localizado no Hospital Universitário, vinculado à Universidade Federal de Sergipe, Brasil. Durante todas as sessões as crianças eram acompanhadas por seus pais ou responsáveis.

\section{Métodos de avaliação}

Com a utilizaçáo do Nintendo Wii ${ }^{\circledast}$ foi criado um avatar com as características reais de cada criança quanto às características físicas, peso, altura e data de nascimento. Foram investigados os dados antropométricos, usando uma fita métrica e uma balança digital modelo Cadence Bal $150^{\circ}$. Esses dados foram registrados na plataforma on-line da Allianz Wordwide Care 2014, que, após estratificação por idade, determinou o índice de massa corporal (IMC).

$\mathrm{Na}$ sequência, foi aplicado o Body Test, que avalia as capacidades físicas, como posição do centro de gravidade, descarga de peso e controle corporal, que refere-se ao processo pelo qual as estruturas do corpo trabalham objetivando o equilíbrio de forças para gerar alinhamento e consequentemente fornecer o equilíbrio.

Foram ainda observados: a percepção corporal através do Teste de Askevold, o teste de Fukuda para equilíbrio corporal e o desempenho nas atividades psicomotoras. Para verificação dessas informaçóes e registro de outros dados foi aplicado um questionário de triagem e avaliação física.

\section{Fase de avaliações}

O trabalho seguiu três fases de investigação, denominadas F1, F2 e F3. Na F1, as crianças passaram por avaliação da percepção corporal, equilíbrio e atividades psicomotoras. $\mathrm{Na}$ fase 2 foram efetuados os 
treinamentos com jogos do Nintendo Wii e na fase 3 foram realizadas as reavaliaçóes.

\section{Percepção corporal}

A percepção corporal, ou esquema corporal, foi avaliada pelo teste de Askevold ${ }^{20}$. Tal avaliação é realizada solicitando que a criança esteja em posiçáo ortostática em frente de uma folha de papel craft colocada na parede a uma altura um pouco superior a da cabeça do voluntário. Em seguida, o pesquisador venda os olhos da criança e emite comandos para que ela toque regióes específicas do corpo. A mesma atividade foi realizada com a criança de olhos abertos. Posteriormente, o pesquisador ligou os pontos marcados no papel, obtendo-se, dessa forma, a área percebida (AP) e a área real (AR). Os pontos demarcados foram:

A - Ponto (1) topo da cabeça: para que se obtém a altura máxima. É aconselhável que seja solicitado apneia inspiratória; na sequência, toca-se o ponto mais alto da cabeça e solicita-se que o avaliado reproduza esse ponto no papel. Isso é realizado três vezes sem que as marcações já registradas sejam vistas.

B - Ponto (2) acrômios direito e esquerdo;

C - Ponto (3) regiáo de maior curvatura da cintura de ambos os lados;

D - Ponto (4) trocânter maior do fêmur bilateralmente.

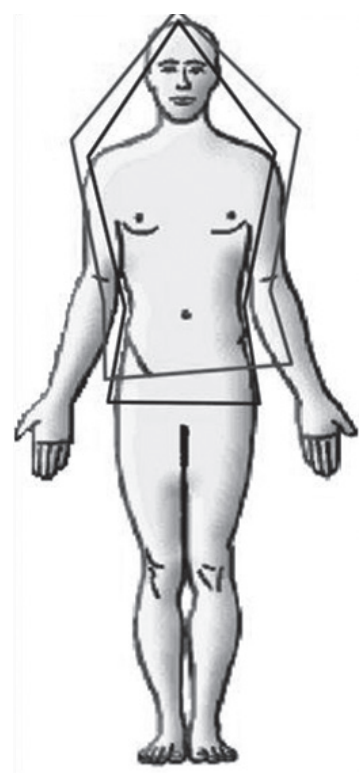

Figura 1: Demonstração esquemática do teste de Askevold. Fonte: Fonseca ${ }^{21}$

Para análise dos resultados obtidos nesse teste foi considerada a área das duas figuras geométricas obtidas a partir da fórmula: $\mathbf{I P C}=\mathbf{A P}-\mathbf{A R} \times \mathbf{1 0 0}$. Em que: $\mathrm{IPC}=$ índice de percepção corporal; $\mathrm{AP}=$ área percebida; $\mathrm{AR}$ = área real (Figura 1).

O valor de referência ideal será considerado como IPC de $100 \%$. Nesse estudo, considerou-se também a severidade da percepção como sendo: $90 \%$ a $100 \%=$ Ótimo; $70 \%$ a $89 \%=$ Bom; $50 \%$ a $69 \%$ = Regular; $30 \%$ a $49 \%$ = Ruim e $\leq 29$ = Péssimo. Para observar o nível de esquema corporal (EC) foram adotados os critérios de Fonseca ${ }^{21}$ e Gama et al..$^{22}$, ou seja, crianças que obtiverem um EC equivalente a $100 \%$ serão consideradas com normoesquematia, acima de $100 \%$ hiperesquematia e abaixo desse valor, hipoesquematia.

\section{Equilíbrio}

Para avaliar o equilíbrio foi realizado o teste de Fukuda. Inicialmente, o avaliador retirou o feedback visual, novamente vendando os olhos da criança e a orientou para estender os braços e realizar marcha elevando os joelhos até a altura do quadril sem se deslocar, executando esse movimento por um período de 1 minuto. Foi considerado alterado ou patológico o deslocamento maior que 1 metro e/ou rotação do corpo superior a $30^{\circ}$.

\section{Atividades psicomotoras}

O desempenho de atividades psicomotoras foi avaliado através do protocolo de teste psicomotor de Fonseca $^{21}$. As atividades são graduadas de $1 \mathrm{a}$ 4, sendo 4 a melhor pontuação. Os itens avaliados foram: equilíbrio estático (apoio retilíneo), equilíbrio dinâmico (marcha controlada), noção do corpo (reconhecimento direita-esquerda), estruturação espaço-temporal (estruturação rítmica), praxia global (coordenação óculo-pedal) e praxia fina (velocidade e precisão).

\section{Protocolo de treinamento com Nintendo Wii}

Foram previamente escolhidos jogos que exigissem coordenação motora, equilíbrio e concentração em uma escala gradual de dificuldade. A realidade virtual foi aplicada duas vezes por semana durante cinco semanas totalizando 10 sessóes de treinamento.

As crianças foram instruídas quanto aos jogos que seriam trabalhados para dar inicio à primeira sessão. Os jogos utilizados foram: Super Hula Hoop e Rhythm Boxing, que estão enquadrados na categoria Aerobics; Tightrope Walk, Soccer Heading, Table Tilt, Penguin Slide e Snowboard Slalom, na categoria Balance; e Snowball 
Fight, Balance Buble e Obstacle Course na categoria Training Plus.

\section{Descrição dos jogos}

O Super Hula Hoop possui duração inicial de três minutos, metade do tempo para a direita e a outra metade para a esquerda, com utilização apenas do Wii Balance Board. Esse jogo estimula o desenvolvimento de certas habilidades, principalmente aquelas relacionadas ao equilíbrio dinâmico (marcha controlada). O Rhythm Boxing tem duração inicial de três minutos, obedecendo aos comandos do treinador virtual. Para realizar esse jogo foram utilizados o Wii Balance Board, controle e Nun-chuck. Ele estimula o desenvolvimento de praxia global (coordenação óculo-manual), praxia global (coordenação óculo-pedal), noção do corpo (reconhecimento direita-esquerda) e estruturação espaço-corporal (estruturação rítmica). Já o Tightrope Walk tem por objetivo chegar ao outro lado do edifício andando por uma corda esticada. Para que a personagem ande, o jogador deve movimentar o corpo para os lados. Utiliza apenas o Wii Balance Board e desenvolve equilíbrio estático (apoio retilíneo), equilíbrio dinâmico (marcha controlada) e praxia global (coordenação óculo-pedal).

No Soccer Heading o jogador é um goleiro, e deve deslocar seu peso de um lado para o outro para defender as bolas e desviar de outros objetos, sendo que cada objeto atingido que não seja uma bola, o personagem perde pontos já conquistados. Utiliza apenas o Wii Balance Board e desenvolve equilíbrio estático (apoio retilíneo), equilíbrio dinâmico (marcha controlada) e praxia global (coordenação óculo-pedal). No Snowball Fight a criança era desafiada durante 120 segundos a realizar movimentos de inclinação lateral do tronco para o lado esquerdo e direito, assim como realizar movimentos com os membros superiores visando acertar um oponente virtual através do lançamento de bolas de neve. O Snowboard Slalom é um jogo em que a pessoa deve simular a descida em uma ladeira coberta de neve, tomando cuidado para passar por entre as setas indicadas durante o percurso. Para obter um bom desempenho se faz necessária uma adequada inclinação anterior e posterior de tronco. Vale ressaltar que para mensurar o desempenho da criança nesse jogo foi considerado apenas o tempo total gasto. Utiliza-se somente o Wii Balance Board para deslocar-se para os lados, para frente e para trás; desenvolve habilidades de equilíbrio estático, noção corporal, agilidade e praxia global (coordenação óculo-pedal).

Através do Table Tilt, é necessário que o jogador conduza bolas para dentro de orifícios situados em uma plataforma instável, que se move para regiōes anteriores e posteriores mediante descargas de peso laterais, fazendo que o centro de gravidade se desloque e mova a bola. Quando todas as bolas são colocadas no orifício da plataforma, progride-se de nível. Com esse jogo, é possível que a criança desenvolva melhores habilidades no equilíbrio estático e controle postural.

Durante o Penguin Slide, a personagem é um pinguim que fica em cima de uma pedra de gelo no oceano, sendo desafiada a capturar a maior quantidade de peixes possível. Promove principalmente o treino de equilíbrio estático, controle corporal e praxia global (coordenação óculo-manual). Jogando o Balance Buble o indivíduo é desafiado a atravessar um rio dentro de uma bolha de ar, devendo ter o máximo de atenção para a bolha não encostar nas margens do rio e estourar. A criança treina o equilíbrio, coordenação motora e lateralidade. Com o Obstacle Course, há simulação de uma caminhada, podendo o jogador aumentar ou reduzir a velocidade e/ou pular para desviar de obstáculos. Utiliza apenas o Wii Balance Board e desenvolve o equilíbrio estático (apoio retilíneo) e equilíbrio dinâmico (marcha controlada).

O Tightrope Walk, Soccer Heading, Snowball Fight e Obstacle Course não associam diretamente o tempo de duração ao nível da atividade, mas há uma contagem de tempo enquanto o jogo está sendo realizado, que se ultrapassada acarreta em perda. Os jogos da categoria Aerobics foram utilizados apenas uma vez, mesmo que a criança não tivesse realizado satisfatoriamente os outros jogos, enquanto os jogos das categorias Balance e Training Plus foram repetidos duas vezes, caso a primeira vez não fosse satisfatória. Em suma, o tempo total de treinamento não ultrapassou 30 minutos.

\section{Reavaliações}

$\mathrm{Na}$ fase pós-intervenção, ou seja, a F3, todas as três crianças foram reavaliadas com os mesmos métodos utilizados na primeira fase.

\section{Análise}

Os resultados desse estudo estão apresentados por meio de dados descritivos das variáveis psicomotoras, realidade virtual e percepção corporal, envolvento dados absolutos dos testes e de frequência expressada por meio de porcentagem. Todos eles foram organizados no programa Microsoft Excel versão de 2010.

\section{Resultados}

As características gerais da amostra estão apresentadas em frequências absolutas e relativas conforme demonstrado na Tabela 1 . 
Na Tabela 2 estão expressos os resultados obtidos nos testes de COG Wii Fit ${ }^{\circ}$, estabilidade corporal e o teste de Fukuda antes e após passarem pelos jogos do Nintendo Wii . Percebeu-se uma melhoria considerável nos três indivíduos na maioria dos testes utilizados. Vale ressaltar que o voluntário três permaneceu com o teste de Fukuda alterado, ou seja, apresentando uma rotação de tronco > $30^{\circ}$ e deslocamento > que $1 \mathrm{~m}$. Esses achados não são considerados normais de acordo com o teste.

Tabela 1: Frequência absoluta e relativa das variáveis apresentadas pela amostra em estudo.

\begin{tabular}{|ccc|}
\hline Variáveis & Número $\left(\mathbf{n}^{\circ}\right)$ & Porcentagem (\%) \\
\hline Gênero & & \\
\hline Masculino & 1 & $33,4 \%$ \\
Feminino & 2 & $66,6 \%$ \\
\hline IMC & & \\
Ideal & 2 & $66,6 \%$ \\
Baixo peso & 0 & $0 \%$ \\
Acima do Peso & 1 & $34,4 \%$ \\
\hline Idade & & \\
\hline 7 a 8 anos & 1 & $34,4 \%$ \\
9 a 10 anos & 2 & $66,6 \%$ \\
\hline
\end{tabular}

Tabela 2: Resultados das avaliações quanto aos testes de COG Wii Fit ${ }^{\circledR}$, estabilidade corporal e o teste de Fukuda antes e após passarem pelos jogos do Nintendo Wii ${ }^{\circledR}$.

\begin{tabular}{|c|c|c|c|c|c|c|}
\hline & \multicolumn{2}{|c|}{ Indiv 1} & \multicolumn{2}{|c|}{ Indiv 1} & \multicolumn{2}{|c|}{ Indiv 1} \\
\hline & Antes & Depois & Antes & Depois & Antes & Depois \\
\hline \multicolumn{7}{|l|}{$\begin{array}{l}\text { COG } \\
\text { Wii Fit }^{\circledast}\end{array}$} \\
\hline MID & $58,2 \%$ & $46,2 \%$ & $49,9 \%$ & $58,1 \%$ & $45,1 \%$ & $54,9 \%$ \\
\hline MIE & $41,8 \%$ & $53,8 \%$ & $43,5 \%$ & $54,2 \%$ & $40,1 \%$ & $50,9 \%$ \\
\hline $\begin{array}{l}\text { Estabili- } \\
\text { dade (\%) }\end{array}$ & $25 \%$ & $80 \%$ & $16 \%$ & $87 \%$ & $15 \%$ & $73 \%$ \\
\hline \multicolumn{7}{|c|}{ Teste de Fukuda } \\
\hline $\begin{array}{l}\text { Desloca- } \\
\text { mento }(\mathrm{cm})\end{array}$ & $1,14 \mathrm{~cm}$ & $90 \mathrm{~cm}$ & $1,22 \mathrm{~cm}$ & $84 \mathrm{~cm}$ & $1,18 \mathrm{~cm}$ & $1,09 \mathrm{~cm}$ \\
\hline $\begin{array}{c}\text { Rotaçáo de } \\
\text { tronco }\end{array}$ & $38^{\circ}$ & $27^{\circ}$ & $45^{\circ}$ & $25^{\circ}$ & $33^{\circ}$ & $32^{\circ}$ \\
\hline \multicolumn{7}{|l|}{ Idade } \\
\hline 7 a 8 anos & & 1 & & & $34,4 \%$ & \\
\hline 9 a 10 anos & & 2 & & & $66,6 \%$ & \\
\hline
\end{tabular}

Legenda: $\mathrm{MID}=$ Membro inferior direito, $\mathrm{MIE}=$ Membro inferior esquerdo. INDIV = Indivíduo. $\mathrm{COG}=$ centro de gravidade. Valores de referência para análise do teste de Fukuda normal $=$ deslocamento $>1 \mathrm{~m}$ e rotação $>$

Os efeitos acumulativos do treinamento de RV ao longo do tempo utilizando o Nintendo Wii podem ser observados na Tabela 3, que apresenta os valores absolutos das atividades utilizadas. Notou-se uma elevação importante nas pontuaçóes alcançadas na maioria dos jogos pelos três voluntários do estudo.

As análises absolutas atribuídas às atividades psicomotoras podem ser visualizadas na Tabela 4. Foi verificado que houve ganhos nas habilidades trabalhadas em todas as crianças incluídas no presente estudo.

Tabela 3: Pontuações absolutas obtidas pelos 3 voluntários em cada atividade do Nintendo $\mathrm{Wii}^{\circledR}$ antes e após as 10 sessões de treinamento.

\begin{tabular}{|c|c|c|}
\hline $\begin{array}{l}\text { Atividades do } \\
\text { Nintendo Wii }\end{array}$ & $\begin{array}{c}\text { Pontuaçáo } \\
\text { Inicial }\end{array}$ & $\begin{array}{c}\text { Pontuaçáo } \\
\text { Final }\end{array}$ \\
\hline \multicolumn{3}{|l|}{ Indivíduo 1} \\
\hline Super Hula Hoop & 50 pontos & 419 pontos \\
\hline Snowball Fight & 20 pontos & 36 pontos \\
\hline Penguin Slide & 44 pontos & 46 pontos \\
\hline Snowboard Slalom & $00: 57.3 \mathrm{~s}$ & $00: 42.2 \mathrm{~s}$ \\
\hline Table Tilt & 30 pontos & 40 pontos \\
\hline Tightrope Walk & 1 ponto & 9 pontos \\
\hline Obstacle Course & 13 pontos & 39 pontos \\
\hline Soccer Heading & 3 pontos & 17 pontos \\
\hline Rhythm Boxing & 171 pontos & 250 pontos \\
\hline Balance Buble & $155 \mathrm{~m}$ & $103 \mathrm{~m}$ \\
\hline \multicolumn{3}{|l|}{ Indivíduo 2} \\
\hline Super Hula Hoop & 158 pontos & 732 pontos \\
\hline Snowball Fight & 12 pontos & 25 pontos \\
\hline Penguin Slide & 49 pontos & 44 pontos \\
\hline Snowboard Slalom & $00: 48.8 \mathrm{~s}$ & $00: 39.7 \mathrm{~s}$ \\
\hline Table Tilt & 0 pontos & 40 pontos \\
\hline Tightrope Walk & 26 pontos & 17 pontos \\
\hline Obstacle Course & 7 pontos & 367 pontos \\
\hline Soccer Heading & 23 pontos & 35 pontos \\
\hline Rhythm Boxing & 161 pontos & 255 pontos \\
\hline Balance Buble & $263 \mathrm{~m}$ & $498 \mathrm{~m}$ \\
\hline \multicolumn{3}{|l|}{ Indivíduo 3} \\
\hline Super HulaHoop & 695 pontos & 827 pontos \\
\hline Snowball Fight & 18 pontos & 24 pontos \\
\hline Penguin Slide & 41 pontos & 64 pontos \\
\hline Snowboard Slalom & $00: 53.2 \mathrm{~s}$ & $00: 40.1 \mathrm{~s}$ \\
\hline Table Tilt & 10 pontos & 10 pontos \\
\hline Tightrope Walk & 10 pontos & 17 pontos \\
\hline Obstacle Course & 1 ponto & 409 pontos \\
\hline Soccer Heading & 9 pontos & 14 pontos \\
\hline Rhythm Boxing & 179 pontos & 234 pontos \\
\hline Balance Buble & $0 \mathrm{~m}$ & $908 \mathrm{~m}$ \\
\hline
\end{tabular}

Legenda: $\mathrm{m}=$ metros; $\mathrm{s}=$ segundos

$\mathrm{Na}$ Figura 2 estão representados os valores do cálculo de percepção corporal segundo o protocolo de Askevold. Por essa classificação é possível identificar como o indivíduo percebe seu corpo no meio em que vive. Notou-se que os três indivíduos avaliados, antes de 
Tabela 4: Pontuações obtidas em todas as atividades psicomotoras das três crianças avaliadas segundo o protocolo de Fonseca de 1995.

\begin{tabular}{lccccc} 
& \multicolumn{1}{c}{$\begin{array}{c}\text { Indiv 1 } \\
\text { Depois }\end{array}$} & Antes & Antes & $\begin{array}{c}\text { Indiv 2 } \\
\text { Depois }\end{array}$ & \multicolumn{2}{c}{ Antes } \\
Depois
\end{tabular}

Legenda: classificaçáo psicomotora $=1$ refere-se a pior pontuação e 4 a melhor.

Percepção corporal

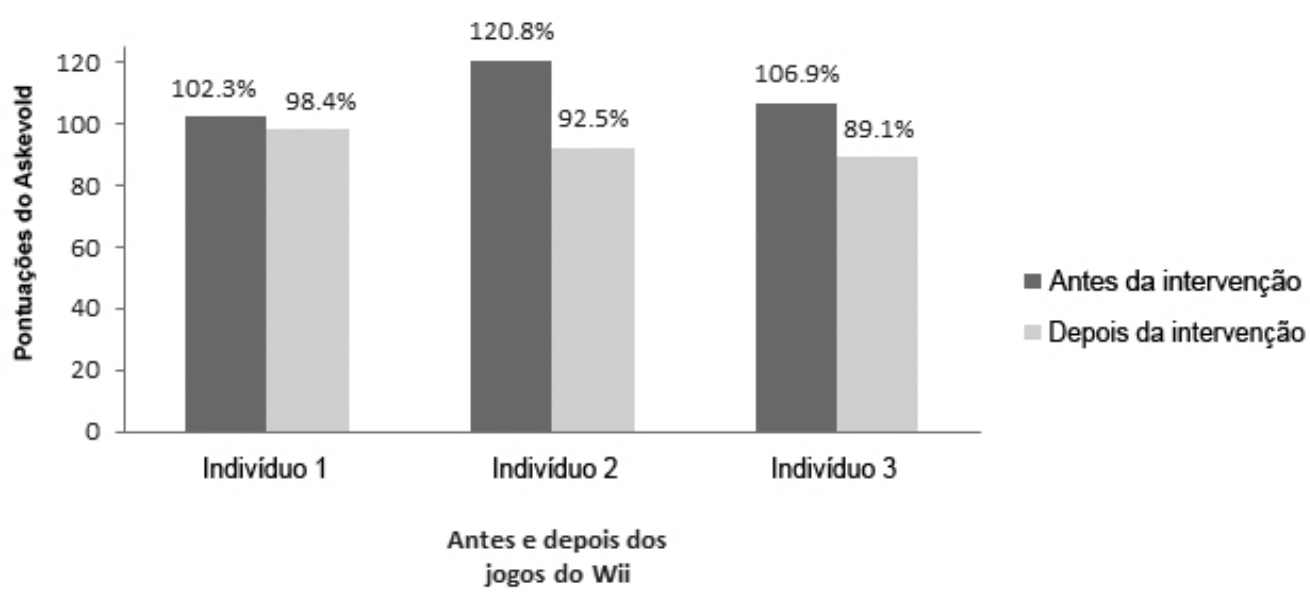

Figura 2: Distribuição percentual das análises do esquema corporal das crianças. Valores de referência = 90\% a 100\% = Ótimo; $70 \%$ a $89 \%$ = Bom; $50 \%$ a $69 \%$ = Regular; 30\% a 49\% = Ruim e $\leq 29$ = Péssimo. Considerou-se no estudo hiperesquematia valores $>$ que $100 \%$.

serem submetidos ao treinamento de realidade virtual apresentavam uma hiperesquematia. Depois das 10 sessões de intervenção apresentaram um esquema corporal considerado ótimo. Nenhum dos indivíduos estudados tiveram hipoesquematia.

\section{Discussão}

Os principais achados desse estudo foram que o Nintendo Wii ${ }^{\odot}$ contribuiu para: (a) melhora considerável da estabilidade e equilíbrio corporal; (b) aperfeiçoamento de habilidades motoras obtidas com a progressão do grau de dificuldade dos jogos e (c) normalização da percepção corporal.

Embora os jogos de realidade virtual estejam sendo amplamente utilizados na infância, os aspectos favoráveis desses videogames ainda não estão suficientemente elucidados. Sobretudo, não há muitas evidências científicas mostrando os efeitos dos jogos de realidade virtual sobre as habilidades psicomotoras e percepção corporal de crianças saudáveis. 
Tem sido sugerido que a utilização de jogos virtuais possa contribuir para o desenvolvimento de habilidades motoras como a coordenação e equilíbrio corporal de indivíduos em fase de reabilitação envolvendo disfunçóes neuromusculoesqueléticas. Um estudo ${ }^{23}$, por exemplo, mostrou que a utilizaçáo do Nintendo Wii em crianças com idade entre seis e dez anos, diagnosticadas com paralisia cerebral espástica, apresentaram ganhos significativos no desempenho motor após 12 semanas de intervenção. Embora sejam crescentes os achados envolvendo populaçóes específicas e/ou com incapacidades, permanece uma lacuna no que concerne aos resultados do uso desses jogos sobre as funçóes psicomotoras de escolares saudáveis ${ }^{24,25}$. Questiona-se também se o uso de jogos virtuais poderia acrescentar ganhos referentes ao desenvolvimento de habilidades psicomotoras e percepção corporal de indivíduos saudáveis.

Vaghetti ${ }^{26}$ demonstrou que Nintendo Wii ${ }^{\oplus}$ foi superior a outros jogos eletrônicos quando os objetivos foram reduzir o sedentarismo, reduzir a obesidade e recuperar movimentos, além de destacar que tal videogame fornece uma boa relação de custo e benefício para os praticantes. Assim como no presente estudo, os testes realizados com o COG Wii Fit realizados por esse autor também identificaram melhora no controle da postura e do equilíbrio.

Segundo Pellegrini ${ }^{27}$, os fatores que são fundamentais para o desenvolvimento motor durante a primeira infância são o equilíbrio e a coordenação. No presente estudo notou-se que tanto o equilíbrio estático como o equilíbrio dinâmico foram melhorados após as crianças serem submetidas ao treinamento com realidade virtual durante as 10 sessóes. Em um estudo realizado em crianças entre quatro e seis anos com dispraxia foram encontrados ganhos significativos relacionados ao equilíbrio, concluindo que além dos jogos promoverem uma interação no ambiente de RV, as intervenções promoviam mudanças benéficas na função motora ${ }^{28}$. No entanto, em estudo de revisão sistemática ${ }^{18}$ verificou que os jogos virtuais não melhoravam o equilíbrio dinâmico. Os autores acreditam que esse tipo de equilíbrio não é sensível o bastante para detectar alteraçóes esperadas na postura. Embora isso tenha sido mostrado, a grande maioria dos autores da comunidade científica apontam para os benefícios oriundos de jogos virtuais, principalmente os mais modernos, em que se faz necessária a plena interação entre os movimentos corporais do participante e o jogo. Tal interação promove adaptaçôes sensoriais e neuromotoras ao longo do tempo, podendo os jogos serem utilizados como uma interface quando o objetivo é promover estimulaçóes referentes ao equilíbrio postural dinâmico e estático de crianças.

Nesse manuscito, o ganho de habilidades referentes ao equilíbrio dinâmico pode ter sido resultado das sucessivas séries utilizando-se jogos com alto grau de exigência motora e raciocínio. O biofeedback e feedforward proporcionados - além dos estímulos somatosensoriais fornecidos - contribuem conjuntamente para sinergismos musculares, adequaçáo dos movimentos articulares, força muscular e aquisição de movimentos mais refinados e elaborados ${ }^{24}$. Além disso, sabe-se que o biofeedback visual proporcionado pelos videogames de fato contribui para a correção postural durante as atividades, proporcionando modulaçóes neuronais capazes de eliminar alteraçóes quanto ao controle motor, evitando as instabilidades ${ }^{18}$. Esse fenômeno também se associa a um estudo que avaliou 29 crianças de ambos os sexos com déficits nos padróes motores, que ao final da intervenção, conseguiram um melhor desempenho nas habilidades relacionadas à estabilidade ${ }^{29}$. Esse achado mostra o quão importante é a utilização de movimentos ou posiçôes específicas para gerar um controle corporal mais eficaz e com isso evoluir aspectos funcionais dos sujeitos.

Em um estudo em que foi utilizada a RV com jogos do Nintendo Wii ${ }^{\circledast}$ em pessoas saudáveis foi perceptível a melhora no equilíbrio e também na força muscular. Embora a força não tenha sido uma variável analisada nesse estudo, a melhora do equilíbrio observada pode ser compreendida também pela maior eficiência de força muscular desenvolvida durante o treinamento com o Nintendo Wii ${ }^{\oplus 24}$.

Com relação às atividades psicomotoras, pôde-se notar que para dois indivíduos não houve ganhos em relação à estruturação espaço-temporal e praxia motora fina, uma vez que as crianças já apresentavam uma pontuação máxima nos testes pré-tratamento. No entanto, em quase todos os indivíduos inseridos na pesquisa observou-se manutenção ou ganhos importantes das habilidades psicomotoras, destacando-se as atividades de praxia óculo-pedal, óculo-manual e noção corporal, além das outras habilidades ora apresentadas. Esse fenômeno pode ser explicado pelo fato das crianças serem desafiadas em cada jogo, sempre buscando ultrapassar seus limites em cada sessão, a fim de obter um novo êxito. Nessa busca, todas as informaçóes vão sendo armazenadas no cérebro, podendo melhorar a qualidade da execução de atividades motoras. Evidências científicas mostram que o nível socioeconômico pode influênciar no desenvolvimento dessas habilidades; crianças com baixo nível socioeconômico exploram objetos e o ambiente livremente, enquanto aquelas cujo padrão de vida é elevado frequentam na maioria das vezes lugares cercados, não explorando de forma livre o ambiente, resultando em prejuízos durante o desenvolvimento de habilidades motoras globais ${ }^{30,31}$.

Nesse estudo, a percepção corporal sofreu alterações notáveis após a submissão do protocolo de realidade 
virtual. $\mathrm{O}$ que chamou atenção foi a regressão das porcentagens do teste de Askevold nos três escolares investigados. Não foram encontrados na literatura estudos que investigassem os efeitos da realidade virtual na percepção corporal de escolares sadios. Isso foi considerado um fator que gerou dificuldades na comparação dos resultados encontrados com trabalhos de outros pesquisadores. No entanto, de acordo com Fonseca ${ }^{21}$ a percepção corporal pode ser melhorada através do aperfeiçoamento dos padróes motores específicos. Tal alternativa pode ser incorporada a um programa de realidade virtual.

Sugere-se que o bom desempenho adquirido na percepção corporal foi decorrente de efeitos adaptativos de controle postural, melhorias na coordenação dos movimentos e aperfeiçoamento do equilíbrio. Esses componentes foram trabalhados quando as crianças eram desafiadas nos jogos preestabelecidos, nos quais o corpo precisava ser movimentado de forma adequada para garantir pontos e avançar de nível. Esse conjunto de estímulos possivelmente modificou o esquema corporal das crianças analisadas.

A principal limitação desse estudo foi a dificuldade na aquisição de um maior número amostral, uma vez que nem todos os pais das crianças se disponibilizaram em levá-las ao lócus da pesquisa. Além disso, a ausência de alguns sujeitos na fase final da investigaçáo contribuiu para perdas no número amostral. Para futuros estudos será fundamental a utilizaçáo dos protocolos de testes e intervençóes nas prórias escolas, em um ambiente previamente organizado para executar as atividades. Essa é uma das alternativas para que haja menos evasão de amostra e facilitar a participação dos escolares. Uma sugestão de possível investigação seria além de avaliar as habilidades motoras e percepção corporal, comparar essas mesmas variáveis com estudantes de diferentes perfis socioeconômicos, ou seja, estudantes de escolas particulares e públicas.

Ao estudar a influência dos jogos de RV sobre as atividades psicomotoras e percepção corporal de escolares sadios dar-se-á contribuição de preencher uma lacuna existente na literatura, visto que essa temática tem sido mais fortemente investigada em indivíduos com lesões neuromusculoesqueléticas, não sendo conhecidos os efeitos desses jogos sobre as atividades motoras e de percepçáo corporal de indivíduos sadios. $\mathrm{O}$ ambiente real simulado por determinados jogos permite à criança a aquisição de habilidades importantes em um ambiente que oferece segurança e aumenta a probabilidade de que essas habilidades sejam transferidas para atividades cotidianas e contribua para a cognição e aprendizagem ${ }^{32}$.

\section{Considerações finais}

Pôde-se constatar que o treinamento com a realidade virtual influenciou positivamente o desempenho nas atividades psicomotoras e percepçáo corporal de todas as crianças analisadas. Foi possível verificar que para a amostra em estudo o uso da tecnologia por meio dos jogos virtuais mostrou-se uma ferramenta importante na aquisição e no fornecimento de estímulos aos sistemas musculoesqueléticos, vestibular e somatossensorial.

\section{Referências}

1. Rossi FS. Considerações sobre a psicomotricidade na educaçáo infantil. Rev Vozes dos Val. 2012;1-18.

2. Almeida C. Perfil psicomotor de alunos com idade entre 7 a 9 anos. $9^{\circ}$ Congresso Nacional de Educaçáo, $3^{\circ}$ Encontro Sul Brasileiro de Psicopedagogia: Anais do $9^{\circ}$ Congresso Nacional de Educação e do $3^{\circ}$ Encontro Sul Brasileiro de Psicopedagogia; 26-29 out. 2009; Curitiba, Brasil. p. 7277-87.

3. Giagazoglou P, Kouliousi C, Sidiropoulou M, Fahantidou A. The effect of institutionalization on psychomotor development of preschool aged children. Res Dev Disabil. Elsevier; 2012;33(3):964-70.

4. Fonseca V. Manual de observação psicomotora: significaçóes psiconeurológicas dos fatores psicomotores. Porto Alegre: Artes Médicas; 1995.

5. Barreto, SJ. Psicomotricidade, educação e reeducação. 2. ed. Blumenau: Livraria Acadêmica; 2000.

6. Rebelatto JR, Castro AP. Equilíbrio estático e dinâmico em indivíduos senescentes e o índice de massa corporal. Fisioter. mov. 2008;21(3):69-75.

7. Cm P, Apqc A, Mg R. Psychomotor development in pre-school with visual deficiency. Pediatr Ther. 2013;3(5):3-6.

8. Lemos LFC, Teixeira LA, Mota CB. Uma revisão sobre centro de gravidade e equilíbrio corporal A review about center of gravity and body balance. Rev. bras. cineantropom. desempenho hum. 2009;17(4):83-90.

9. Hsu Y, Kuan C, Young Y. Assessing the development of balance function in children using stabilometry. Int J Pediatr Otorhinolaryngol Extra. 2009;73:737-40.

10. Arruda, KMF; Silva EAA. Desenvolvimento motor na educação infantil através da ludicidade. Connection online [periódico na Internet]. 2009 [acesso em 2016 Mai 22];(4):3750. Disponível em: http://www.periodicos.univag.com.br/ index.php/CONNECTIONLINE/article/view/131/363

11. Turtelli LS. Caminhos da pesquisa em imagem corporal na sua relaçáo com o movimento. Rev. bras. ciênc. esporte. 2002;24(1):151-66.

12. Pinquart M. Body image of children and adolescents with chronic illness: A meta-analytic comparison with healthy peers. Body Image. Elsevier; 2013;10(2):141-8.

13. Lewer M, Nasrawi N, Schroeder D, Vocks S. Body image disturbance in binge eating disorder: a comparison of obese patients with and without binge eating disorder regarding the cognitive, behavioral and perceptual component of body image. Eat Weight Disord. 2015;21(1):115-25. 
14. Hartmann AS, Thomas JJ, Greenberg JL, Elliott CM, Matheny NL, Wilhelm S. Anorexia nervosa and body dysmorphic disorder: a comparison of body image concerns and explicit and implicit attractiveness beliefs. Body Image. Elsevier; 2015;14:77-84.

15. Segheto W, Segheto KJ, Borim C, Gama F. Proposta de categorização para análise da percepção corporal.

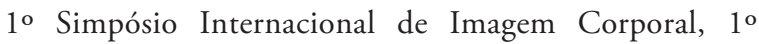
Congresso Brasileiro de Imagem Corporal: Anais do

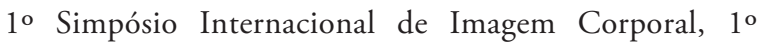
Congresso Brasileiro de Imagem Corporal; 9-10 ago. 2010; Campinas, Brasil. p. 1-7.

16. Ferreira AAR. A importância da psicomotricidade na escola [monografia]. Rio de Janeiro: Universidade Cândido Mendes; 2003.

17. Monteiro CBM. Realidade virtual na paralisia cerebral. São Paulo: Plêiade; 2011.

18. Sobral R, Junior M. Efetividade da reabilitação virtual no equilíbrio corporal e habilidades motoras de indivíduos com déficit neuromotor: uma revisão sistemática. Rev bras ativ fís saúde. 2012;17(3):224-30.

19. Goble DJ, Cone BL, Fling BW. Using the Wii Fit as a tool for balance assessment and neurorehabilitation: the first half decade of "Wii-search". J Neuroeng Rehabil. 2014;11(1):1-9.

20. Askevold F. Measuring body image. Psychoter Psychosom. 1975;26(2):71-7.

21. Fonseca, CC. Análise do esquema corporal e imagem corporal na dança de saláo e seus aspectos motivacionais [dissertação]. São Paulo, Brasil: Universidade São Judas Tadeu; 2008.

22. Gama EF, Dantas DB, Almeida ET \& Thurm BE. Influência da natação na percepção corporal. Rev bras ciênc mov. 2009; 17(2),1-18.
23. Alsaif AA, Alsenany S. Effects of interactive games on motor performance in children with spastic cerebral palsy. J Phys Ther Sci. 2015; 27(6):2001-03.

24. Schiavinato AM, Baldan C, Melatto L, Lima LS, Barthel D, Lawton D. Influência do Wii Fit no equilíbrio de paciente com disfunção cerebelar: estudo de caso. J Heal Sci Inst. 2010;28(1):50-2.

25. Ting LH. Dimensional reduction in sensorimotor systems: a framework for understanding muscle coordination of posture. Prog Brain Res. 2007;165:299-321.

26. Vaghetti CAO, Botelho SSC. Ambientes Virtuais de Aprendizagem na Educação Física: uma revisão sobre a utilização de exergames. Ciênc cogn. 2010;15(1):76-88.

27. Pellegrini AM, Neto SS, Bueno FCR, Alleoni BN, Motta AI. Desenvolvendo a coordenaçáo motora no ensino fundamental. In: Pinho SZ, Saglietti JRC, organizadores. Núcleos de ensino. São Paulo: Unesp; 2003.

28. Ashkenazi T, Weiss PL, Orian D, Laufer Y. Low-cost virtual reality intervention program for children with developmental coordination disorder: a pilot feasibility study. Pediatr Phys Ther. 2013;25(4):467-73.

29. Mombarg R, Jelsma D, Hartman E. Effect of Wiiintervention on balance of children with poor motor performance. Res Dev Disabil. Elsevier; 2013;34(9):2996-3003.

30. Neto AS, Mascarenhas LPG, Nunes GF, Lepre C, Campos W. Relação entre fatores ambientais e habilidades motoras básicas em crianças de 6 e 7 anos. 2004;3(3):135-40.

31. Murijo MG, Pereira K. Perfil psicomotor na Praxia Global e Fina de crianças de três a cinco anos pertencentes à escola privada e pública. Conscientiae saúde. 2008;7(2):151-7.

32. Wang M, Reid D. virtual reality in pediatric neurorehabilitation: attention deficit hyperactivity disorder, autism and cerebral palsy. Neuroepidemiology. 2011;36(1):2-18.

\section{Como citar este artigo:}

Souza EC, Ferreira APL. Realidade virtual e atividades psicomotoras. Rev. Aten. Saúde. 2016;14(48):11-20. 